\title{
Typische Beschwerden von Musikern
}

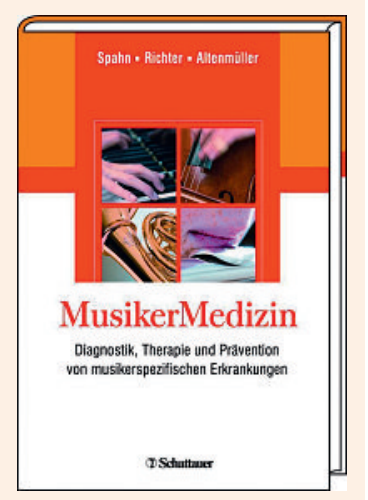

Claudia Spahn, Bernhard Richter, Eckart Altenmüller (Hrsg.) MusikerMedizin

Diagnostik, Therapie und Prävention von musikerspezifischen Erkrankungen.

Stuttgart: Schattauer; 2010.

416 Seiten, 126 Abbildungen, $109 \mathrm{CHF}$.

ISBN 978-3-7945-2634-5
Musikermedizin ist ein eigenständiges, wenn auch noch sehr junges Fachgebiet. Die Musikermedizin untersucht typische Beschwerdebilder von Musikern und sucht nach Therapieansätzen. Die körperliche und psychische Belastung eines Berufsmusikers oder Sängers ähnelt in vielerlei Hinsicht der eines Leistungssportlers. Es war an der Zeit, ein fundiertes Buch über die medizinischen Aspekte des Musizierens, die Diagnostik, Therapie und Prävention von musikerspezifischen Erkrankungen zu schreiben.

Das Bewusstsein für einen Bedarf an musikerspezifischen Kenntnissen geht in das frühe letzte Jahrhundert zurück. In den achtziger Jahren wurde in Deutschland die Deutsche Gesellschaft für Musikphysiologie und Musikmedizin (DGfMM) gegründet. Verschiedene Publikationen über die Belastung von Orchestermusikern, Solisten und Sängern folgten. Zum Vergleich: Die Deutsche Gesellschaft für Sportmedizin und Prävention wurde schon 1912 gegründet. In Hannover wurde 1974 das Institut für Musikphysiologie und Musikermedizin eröffnet, in Berlin 2002 das Kurt-Singer-Institut und in Freiburg 2005 das Freiburger Institut für Musikmedizin. Die drei Herausgeber des Werkes sind nicht nur Mediziner, sondern auch professionelle und passionierte Musiker. Frau Prof. Claudia Spahn ist Leiterin des Freiburger Instituts für Musikermedizin (FMI). Bernhard Richter ist Professor für Musikermedizin am Freiburger Institut und Leiter des Zentrums für Musikermedizin. Eckart Altenmüller ist Professor für Musikermedizin an der Hochschule für Musik und Leiter des Instituts für Musikphysiologie und Musikermedizin in Hannover.

In der Musikermedizin spielen Prävention und Gesundheitsförderung eine entscheidende Rolle. Musiker klagen häufig über Überlastungssyndrome des Bewegungsapparates, über neurologische Beschwerden durch repetitive Bewegungsabläufe oder über Auftrittsängste. Diese spezifischen Anforderungen werden in dem Werk umfassend berücksichtigt und praxisnah präsentiert. Spezielle Erkrankungen werden jeweils mit Fallbeispielen aus der Musikersprechstunde untermauert. Das Buch ist in zwei Hauptteile gegliedert: Der erste informiert über die fächerübergreifenden Grundlagen. In der Einführung wird die historische Entwicklung bis zur aktuellen Situation dargestellt, die Bedeutung evaluiert, und es werden Hinweise zu Ausbildungen gegeben. Die folgenden Kapitel befassen sich mit Epidemiologie, Diagnostik, Therapie und Prävention.

Im zweiten Hauptteil über Diagnostik und Therapie verschiedener medizinischer Fachgebiete werden Musikererkrankungen und -syndrome vorgestellt. Während man in Bezug auf Erkrankungen von Musikern vorwiegend an HNO, Orthopädie und Neurologie denkt, sollten Dermatologie, Augenheilkunde und das grosse Gebiet der Inneren Medizin nicht vergessen werden. In dem umfassenden Werk kommen all diese und sogar noch weitere jedenfalls nicht zu kurz.

Sandra Krüger, Berlin 